\title{
Bei leichter Bronchiolitis hilft jede Inhalationstherapie
}

Anil AB et al. High Volume Normal Saline Alone Is as Effective as Nebulized Salbutamol-Normal Saline, Epinephrine-Normal Saline, and 3\% Saline in Mild Bronchiolitis.

Pediatr Pulmonol. 2010;45:41-47

\section{Hintergrund}

Bis zum Alter von drei Jahren haben alle Kinder eine Atemwegsinfektion mit Respiratory-Syncytial-Viren (RSV) durchgemacht. Etwa 2-3\% der unter Einjährigen müssen wegen einer saisonalen, RSV-induzierten Bronchiolitis ins Krankenhaus aufgenommen werden.

Effiziente Regimes zur Behandlung einer RSV-induzierten Bronchiolitis sind rar. Inhalative Behandlungen mit Bronchodilatatoren, Epinephrin, topischen oder systemischen Steroiden werden vielfach angewendet. Signifikante Effekte wurden für die Behandlung mit einer dreiprozentigen hypertonen Kochsalzlösung bei hospitalisierten Säuglingen mit Bronchiolitis belegt.

\section{Fragestellung}

Anil und Kollegen untersuchten, welche Therapie bei milder Bronchiolitis im ambulanten Bereich wirksam ist.

\section{Methodik}

In die prospektive, randomisierte, doppelblinde, kontrollierte Studie wurden Säuglinge im Alter zwischen sechs und 24 Monaten aufgenommen, die erstmals eine Bronchiolitis hatten und in der Notfallambulanz vorgestellt wurden. Die Bronchiolitis war bei allen Säuglingen mild (klinischer Schweregrad $\leq 9$ bei den Kriterien Atemfrequenz, Giemen, Einziehungen, Allgemeinzustand; jeweiliges Maximum = 3).

Die Behandlung erfolgte durch die zweimalige Inhalation von je $4 \mathrm{ml}$ der zu testenden Inhalationslösung mithilfe eines Medic-Aid-Sidestream-Nebulizers über eine Gesichtsmaske bei einem Fluss von $61 / \mathrm{min}$, betrieben mit $100 \%$ Sauerstoff.

Es wurden fünf verschiedene Inhalationslösungen verglichen:
_Gruppe 1: 1,5 mg Adrenalin in $4 \mathrm{ml}$ $\mathrm{NaCl}(0,9 \%)$,

-Gruppe 2: 1,5 mg Adrenalin in $4 \mathrm{ml}$ $\mathrm{NaCl}(3 \%)$,

_ Gruppe 3: 2,5 mg Salbutamol in $4 \mathrm{ml}$ $\mathrm{NaCl}(0,9 \%)$,

- Gruppe 4: 2,5 mg Salbutamol in $4 \mathrm{ml}$ $\mathrm{NaCl}(3 \%)$,

-Gruppe 5: 0,9\% NaCl.

\section{Ergebnisse}

Der klinische Schweregrad lag zu Beginn der Inhalationstherapie im Mittel zwischen 3,5 und 4,1 und fiel dann linear auf Werte zwischen 1,6 und 2,3 nach zwei Stunden ab. Die Sauerstoffsättigung und die Herzfrequenz blieben in etwa konstant bei $98 \%$ bzw. $135 / \mathrm{min}$.

\section{Schlussfolgerung}

Die verglichenen Regimes zeigten keinen Unterschied hinsichtlich des Therapieerfolgs. Zu beachten ist, dass die Ergebnisse nur für Kinder mit leichter Bronchiolitis und kurzer Therapiedauer gelten.

\section{Kommentar}

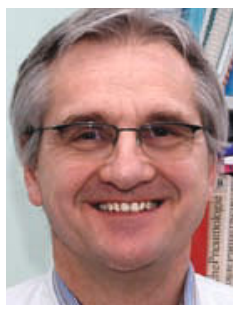

Prof. Dr. med. Matthias Griese, München

Die Ergebnisse sind extrem limitiert: Das Fehlen einer Placebogruppe macht Aussagen zum Spontanverlauf unmöglich. Die nur mäßige Fallzahl erlaubt nur, Unterschiede von mehr als $55 \%$ zu identifizieren.

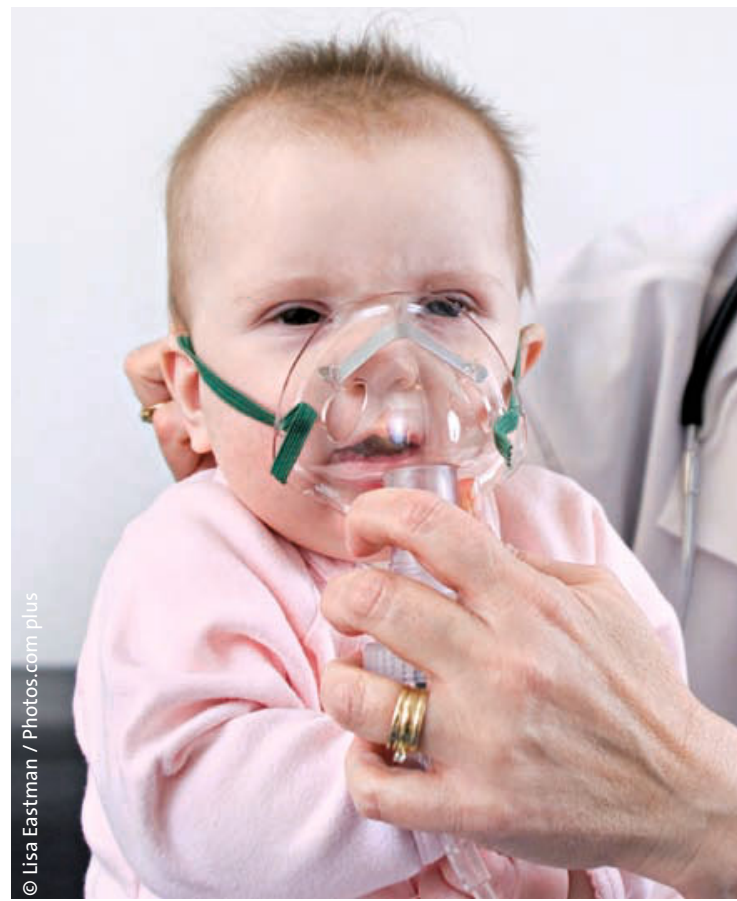

Säuglinge mit milder Bronchiolitis sprechen gut auf eine Inhalationstherapie an.

Da die Verbesserungen in allen Gruppen etwa in dieser Größenordnung lagen, waren geringere Unterschiede, wie sie z.B. für eine hypertone Kochsalzlösung bei hospitalisierten Kindern nachgewiesen wurden, nicht zu finden.

\section{Fazit}

Die Untersuchung zeigt, dass die milde Bronchiolitis des Säuglings im Kurzzeitverlauf sehr gut auf eine Inhalationstherapie gleich welcher Art anspricht. Ob sie besser ist als keine Inhalationstherapie, lässt sich aufgrund des Fehlens der Placebogruppe nicht feststellen. Der langfristige Verlauf zeigte jedoch in allen Gruppen eine gleich hohe stationäre Aufnahmerate von ca. $20 \%$. Dies könnte als eine entweder im Verlauf fehlende Therapie oder eine nicht ausreichend nachhaltig wirksame Behandlung der Kinder interpretiert werden.

Damit zeigt sich, dass wir dringend große Untersuchungen über ausreichend lange Zeiträume und unter Einschluss aller relevanten Kontrollgruppen, einschließlich Placebo und Nichtbehandlung, für dieses sehr häufige klinische Problem benötigen. 